\title{
New Critical Point Induced by the Axial Anomaly in Dense QCD
}

\author{
Tetsuo Hatsuda, ${ }^{1}$ Motoi Tachibana, ${ }^{2}$ Naoki Yamamoto ${ }^{1}$ and Gordon Baym ${ }^{3}$ \\ ${ }^{1}$ Department of Physics, University of Tokyo, Japan \\ ${ }^{2}$ Department of Physics, Saga University, Saga 840-8502, Japan \\ ${ }^{3}$ Department of Physics, University of Illinois, 1110 W. Green St., Urbana, Illinois 61801
}

\begin{abstract}
We study the interplay between chiral and diquark condensates within the framework of the Ginzburg-Landau free energy, and classify possible phase structures of two and three-flavor massless QCD. The QCD axial anomaly acts as an external field applied to the chiral condensate in a color superconductor and leads to a crossover between the broken chiral symmetry and the color superconducting phase, and, in particular, to a new critical point in the QCD phase diagram.
\end{abstract}

PACS numbers: 12.38.-t,12.38.Mh,26.60.+c

Quantum chromodynamics at finite temperature, $T$, and chemical potential, $\mu$, exhibits a rich phase structure, from the hadronic Nambu-Goldstone (NG) phase at low $T$ and $\mu$, to the quark-gluon plasma (QGP) at high $T$, and color superconductivity (CSC) 1] at high $\mu$. The phase transition from the NG phase to the QGP is being studied experimentally in ultrarelativistic heavy ion collisions at RHIC, and will be in the future at the LHC 2]. The transition from the NG to the CSC phase could also be relevant in neutron and quark stars.

The properties of the phases of dense matter in QCD depend on an important interplay between two competing phenomena: quark-antiquark pairing, characterized by a chiral condensate $\langle\bar{q} q\rangle$, and quark-quark pairing, characterized by a diquark condensate $\langle q q\rangle[\underline{3}]$. We investigate here how this interplay determines the transition between the NG and the CSC phases, via a modelindependent Ginzburg-Landau (GL) approach in terms of two order-parameter fields, the chiral condensate $\Phi$ and diquark condensate $d$. This phenomenon is interesting not only in its own right, but also to other systems, e.g. the interplay between magnetically ordered phases and metallic superconductivity [4].

We consider two simple but non-trivial cases, 3-flavor quark matter with equal numbers of massless up (u), down (d), and strange (s) quarks, and 2-flavor quark matter with equal numbers of massless $\mathrm{u}$ and $\mathrm{d}$ quarks. Chiral and color symmetries rather stringently constrain the possible couplings between $\Phi$ and $d$. As we show, the $\Phi$ - $d$ coupling induced by the axial anomaly leads to a crossover between the NG phase and the CSC phase and also to a new critical point in the QCD phase diagram. The former may be relevant to the question of continuity of the hadronic and quark matter [5]. The case of two light quarks ( $\mathrm{u}$ and $\mathrm{d}$ ) and one medium-heavy (s) quark in $\beta$-equilibrium with charge neutrality will be reported elsewhere.

The GL free energy in three spatial dimensions in massless three-flavor QCD is $\Omega=\Omega_{\chi}+\Omega_{d}+\Omega_{\chi d}$. The color-singlet chiral field $\Phi_{i j} \sim-\left\langle\bar{q}_{R}^{j} q_{L}^{i}\right\rangle$ is described by the standard free-energy [6]:

$$
\begin{aligned}
\Omega_{\chi}= & \frac{a_{0}}{2} \operatorname{Tr} \Phi^{\dagger} \Phi+\frac{b_{1}}{4 !}\left(\operatorname{Tr} \Phi^{\dagger} \Phi\right)^{2}+\frac{b_{2}}{4 !} \operatorname{Tr}\left(\Phi^{\dagger} \Phi\right)^{2} \\
& -\frac{c_{0}}{2}\left(\operatorname{det} \Phi+\operatorname{det} \Phi^{\dagger}\right)
\end{aligned}
$$

where "Tr" and "det" are taken over the flavor indices, $i$ and $j$. Under $S U(3)_{L} \times S U(3)_{R} \times U(1)_{B} \times U(1)_{A}$ rotations, the chiral field transforms as $\Phi \rightarrow \mathrm{e}^{2 i \alpha_{A}} V_{L} \Phi V_{R}^{\dagger}$ where the phase $\alpha_{A}$ is associated with the $U(1)_{A}$ rotation. The first three terms on the right of Eq. (11) are invariant under this rotation; the fourth term, caused by the axial anomaly, breaks the $U(1)_{A}$ symmetry down to $Z_{2 N_{f}}=Z_{6}$. We assume that $c_{0}>0$, a necessary condition for the $\eta^{\prime}$ mass to obey $m_{\eta^{\prime}}^{2}>0$ for positive $\Phi$. We also assume that the chiral phase transition is driven by $a_{0}$ changing sign. The cubic determinant term in $\Omega_{\chi}$ makes the chiral transition with three flavors first order.

We focus on Lorentz scalar diquarks belonging to the fundamental representations in color and flavor space: $\left\langle\left(q_{L}\right)_{b}^{j} C\left(q_{L}\right)_{c}^{k}\right\rangle \sim \epsilon_{a b c} \epsilon_{i j k}\left[d_{L}^{\dagger}\right]_{a i}$ and $\left\langle\left(q_{R}\right)_{b}^{j} C\left(q_{R}\right)_{c}^{k}\right\rangle \sim$ $\epsilon_{a b c} \epsilon_{i j k}\left[d_{R}^{\dagger}\right]_{a i}$ where $i, j, k(a, b, c)$ are the flavor (color) indices, and $C$ is the charge conjugation matrix. Un$\operatorname{der} S U(3)_{L} \times S U(3)_{R} \times U(1)_{B} \times U(1)_{A} \times S U(3)_{C} \equiv \mathcal{G}$, $d_{L, R}$ transforms as $d_{R} \rightarrow e^{2 i\left(\alpha_{B}+\alpha_{A}\right)} V_{R} d_{R} V_{C}^{\mathrm{t}}, d_{L} \rightarrow$ $e^{2 i\left(\alpha_{B}-\alpha_{A}\right)} V_{L} d_{L} V_{C}^{\mathrm{t}}$; then $\left(d_{R} d_{L}^{\dagger}\right) \rightarrow e^{4 i \alpha_{A}} V_{R}\left(d_{R} d_{L}^{\dagger}\right) V_{L}^{\dagger}$. The most general form of the GL free energy of the $d$ field, to $\mathcal{O}\left(d^{4}\right)$, is $[7,8,9]$ :

$$
\begin{aligned}
\Omega_{d} & =\alpha_{0} \operatorname{Tr}\left[d_{L} d_{L}^{\dagger}+d_{R} d_{R}^{\dagger}\right] \\
& +\beta_{1}\left(\left[\operatorname{Tr}\left(d_{L} d_{L}^{\dagger}\right)\right]^{2}+\left[\operatorname{Tr}\left(d_{R} d_{R}^{\dagger}\right)\right]^{2}\right) \\
& +\beta_{2}\left(\operatorname{Tr}\left[\left(d_{L} d_{L}^{\dagger}\right)^{2}\right]+\operatorname{Tr}\left[\left(d_{R} d_{R}^{\dagger}\right)^{2}\right]\right) \\
& +\beta_{3} \operatorname{Tr}\left[\left(d_{R} d_{L}^{\dagger}\right)\left(d_{L} d_{R}^{\dagger}\right)\right]+\beta_{4} \operatorname{Tr}\left(d_{L} d_{L}^{\dagger}\right) \operatorname{Tr}\left(d_{R} d_{R}^{\dagger}\right)
\end{aligned}
$$

This free energy is invariant under $\mathcal{G}$. We assume that the normal-CSC transition is driven by $\alpha_{0}$ changing sign. Since $d_{L, R}$ carries baryon number, $\operatorname{det} d_{L, R}$ terms are not allowed, unlike for $\Phi$.

The interaction free energy of the chiral and diquark 
fields is, to fourth order,

$$
\begin{aligned}
\Omega_{\chi d}= & \gamma_{1} \operatorname{Tr}\left[\left(d_{R} d_{L}^{\dagger}\right) \Phi+\left(d_{L} d_{R}^{\dagger}\right) \Phi^{\dagger}\right] \\
& +\lambda_{1} \operatorname{Tr}\left[\left(d_{L} d_{L}^{\dagger}\right) \Phi \Phi^{\dagger}+\left(d_{R} d_{R}^{\dagger}\right) \Phi^{\dagger} \Phi\right] \\
& +\lambda_{2} \operatorname{Tr}\left[d_{L} d_{L}^{\dagger}+d_{R} d_{R}^{\dagger}\right] \cdot \operatorname{Tr}\left[\Phi^{\dagger} \Phi\right] \\
& +\lambda_{3}\left(\operatorname{det} \Phi \cdot \operatorname{Tr}\left[\left(d_{L} d_{R}^{\dagger}\right) \Phi^{-1}\right]+\text { h.c. }\right) .
\end{aligned}
$$

The triple boson coupling $\sim \gamma_{1}$, which breaks the $U(1)_{A}$ symmetry down to $Z_{6}$, originates from axial anomaly. The remaining terms are fully invariant under $\mathcal{G}$ [10. Equations (11)-(3) constitute the most general form of the GL free energy under the conditions that the phase transition is not strongly first order and that the condensed phases are spatially homogeneous.

We assume, for three flavors in the chiral limit, a flavor symmetric chiral condensate, $\Phi=\operatorname{diag}(\sigma, \sigma, \sigma)$, and a color-flavor-locked (CFL) diquark condensate, $d_{L}=$ $-d_{R}=\operatorname{diag}(d, d, d)$, in which all flavors contribute equally to the $J^{P}=0^{+}$pairing. Then the GL free-energy, the sum of Eqs. (11)-(3), reduces to

$$
\begin{aligned}
\Omega_{3 F}= & \left(\frac{a}{2} \sigma^{2}-\frac{c}{3} \sigma^{3}+\frac{b}{4} \sigma^{4}\right)+\left(\frac{\alpha}{2} d^{2}+\frac{\beta}{4} d^{4}\right) \\
& -\gamma d^{2} \sigma+\lambda d^{2} \sigma^{2} .
\end{aligned}
$$

Because the cubic $d^{2} \sigma$ and $\sigma^{3}$ terms in Eq. (4) both arise from the axial anomaly, $\gamma$ and $c$ are related microscopically. Indeed, it is straightforward to show from the instanton-induced six-fermion interaction, $\operatorname{det}_{i, j}\left(\bar{q}_{R}^{j} q_{L}^{i}\right)$, that $\gamma$ has the same sign and the same order of magnitude as $c$ 11, 12]. Positive $\gamma$ (attraction) favors coexistence, $\sigma \neq 0, d \neq 0$. Also, the $d^{2} \sigma$-term acts to break chiral symmetry explicitly, implying that $\sigma \neq 0$ may be realized for all baryon densities, as discussed below.

As we can show from microscopic calculations in weak coupling QCD and in the NJL model, $\lambda>0$ and $\beta>0$. A non-vanishing $\sigma$ plays the role of an effective mass for the quark field, reducing the density of states at the Fermi surface, and the pairing energy [9], an effect represented $\lambda d^{2} \sigma^{2}>0$. Furthermore, we find $\lambda / \beta \sim \ln \left(\Lambda / T_{d}\right) /\left(\mu / T_{d}\right)^{2}$, which is rather small for reasonable values of $\mu, T_{d}$ (the critical temperature of the color superconductivity without the $\sigma-d$ coupling), and $\Lambda$ ( $\sim \mu$ for weak coupling QCD, and in the NJL model, $\sim$ the spatial momentum cutoff). We consider here the first order chiral transition driven by $c$ with positive $b$; the case with negative $b$ in three flavors does not change the results qualitatively [12]. On the basis of the above discussion, we focus on the case $\gamma>0, \lambda \geq 0$ with $b>0$.

In principle, the system can have four possible phases: normal (NOR) with $\sigma=d=0, \operatorname{CSC}(\sigma=0, d \neq 0)$, NG $(\sigma \neq 0, d=0)$, and coexistence (COE) $(\sigma \neq 0, d \neq 0)$. We locate the phase boundaries and the order of the phase transitions by comparing the free energies, $\Omega^{(\mathrm{NOR})}(0,0)$, $\Omega^{(\mathrm{CSC})}(0, d), \Omega^{(\mathrm{NG})}(\sigma, 0)$, and $\Omega^{(\mathrm{COE})}(\sigma, d)$.

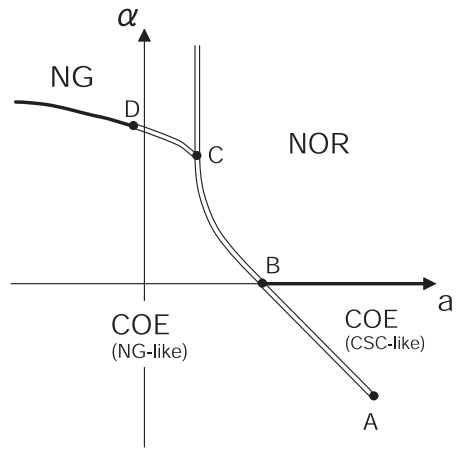

FIG. 1: Phase structure in the three-flavor system with $\gamma>0$ and $\lambda=0$. The phase boundaries with a first (second) order transition is denoted by a double (single) line.

In the COE phase, it is useful to analyze the free energy in terms of the single variable, $\sigma(d)$, or $d(\sigma)$, obtained by solving the stationarity condition, $\partial \Omega^{(\mathrm{COE})}(\sigma, d) / \partial d=0$.

In the absence of $\sigma-d$ coupling $(\gamma=\lambda=0)$, the four phases defined above are separated by $\alpha=0$ (a line of second order transitions) and by $a=2 c^{2} /(9 b)$ (a line of first order phase transitions). But with an attractive coupling, $\gamma>0$ with $\lambda=0$, the phase structure undergoes four major modifications, as shown in Fig [ where the first and second order phase boundaries are shown by double and single lines respectively: (i) The area of the COE phase grows, since the $d^{2} \sigma$ term lowers the free energy if both $\sigma$ and $d$ are nonvanishing. (ii) The first order line between the CSC-like and the NG-like COE, originally located at $a=2 c^{2} /(9 b)$, terminates at a critical point, A [13]. This behavior is expected since $d^{2} \sigma$ acts as an external field for $\sigma$, washing out the first order phase transition for sufficiently large $\gamma$ or $d$. The NG-like COE phase in Fig. 11 1 has larger $\sigma$ than the CSC-like COE phase across the boundary BA. (iii) The second order boundary originally at $\alpha=0$ splits in two, a line going to the right from the critical end point $\mathrm{B}$ and a line going to the left from the point C. Since $\sigma$ changes discontinuously across the first order boundary CB, the $d^{2} \sigma$ term, which acts as a mass term for $d^{2}$, leads to different critical temperatures for diquark condensation to the NG-like COE and CSC-like COE. (iv) For $\gamma>(\sqrt{\beta / b}) c / 3$, a tricritical point $\mathrm{D}$ appears on the phase boundary between NG and COE. Then the point C, otherwise a critical end point, becomes a triple point.

Table I locates the characteristic points, A-D, in the $(a, \alpha)$ plane. To show explicitly how the critical point A appears, we derive an effective free-energy $\Omega_{3 F}[\sigma, d(\sigma)]$, using the stationarity condition, $d^{2}=2(\gamma \sigma-\alpha / 2) / \beta$ :

$$
\Omega_{3 F}[\sigma, d(\sigma)]=-\frac{\alpha^{2}}{4 \beta}+\alpha^{*} \sigma+\frac{a^{*}}{2} \sigma^{2}-\frac{c}{3} \sigma^{3}+\frac{b}{4} \sigma^{4},
$$

with $\alpha^{*} \equiv \alpha \gamma / \beta$ and $a^{*} \equiv a-2 \gamma^{2} / \beta$. Equation (5) is valid for $\sigma \geq \alpha /(2 \gamma)$. We eliminate the $\sigma^{3}$ term in 
Eq. (15), by introducing $\tau=\sigma-c /(3 b)$. Then the system becomes equivalent to an Ising ferromagnet in an external magnetic field. The point A in Fig 1 corresponds to the second order critical point of this magnetic system.

The $\lambda d^{2} \sigma^{2}$ term, with positive $\lambda$, modifies the coefficients $a^{*}, c$ and $b$ in Eq. (5) as $a^{*} \rightarrow a^{*}-2 \alpha \lambda / \beta$, $c \rightarrow c-6 \gamma \lambda / \beta$ and $b \rightarrow b-4 \lambda^{2} / \beta$. Therefore, for $\lambda / \beta \ll 1$, as suggested microscopically, the $\lambda$ term does not qualitatively change the phase diagram in Fig 1

Let us discuss the axial anomaly-driven crossover from the point of view of chiral symmetry. The CSC phase with a CFL structure $\left(d_{L} d_{R}^{\dagger}=-\operatorname{diag}\left(d^{2}, d^{2}, d^{2}\right) \neq 0\right)$ breaks chiral symmetry but preserves the $Z_{4}$ discrete subgroup of $U(1)_{A}$. On the other hand, in the COE phase, $d_{L} d_{R}^{\dagger}=-\operatorname{diag}\left(d^{2}, d^{2}, d^{2}\right) \neq 0$ and $\Phi=\operatorname{diag}(\sigma, \sigma, \sigma)$ lead to chiral symmetry breaking, preserving only $Z_{2}$. The symmetry breaking pattern is different in the two phases. However, the $\gamma_{1}$-term in Eq. (3) has $Z_{6}$ symmetry which contains $Z_{2}$ (but not $Z_{4}$ ) as a subgroup. Therefore, once the axial anomaly is present, the NG-like and CSC-like COE phases cannot be distinguished by symmetry and can be continuously connected. The NG and COE phases differ in the realization of $U(1)_{B}$ symmetry, and therefore their boundary is not smoothed out.

We turn now to the massless two-flavor system (with infinite s quark mass). In this case, all chiral and diquark condensates with an s quark are suppressed; we write $\Phi=\operatorname{diag}(\sigma, \sigma, 0)$ and $d_{L}=-d_{R}=\operatorname{diag}(0,0, d)$. The latter is the two flavor color superconductivity, 2SC state. Due to this color-flavor structure, the cubic terms in $\sigma$ and $d$ are identically zero, and the model reduces to:

$\Omega_{2 F}=\left(\frac{a}{2} \sigma^{2}+\frac{b}{4} \sigma^{4}+\frac{f}{6} \sigma^{6}\right)+\left(\frac{\alpha}{2} d^{2}+\frac{\beta}{4} d^{4}\right)+\lambda d^{2} \sigma^{2} .(6)$

Since in two flavor QCD at finite $T$ and $\mu$, a tricritical point $(a=b=0)$ may exist at which the second order transition for $b>0$ turns into a first order transition for $b<0$ [15], we introduce a $\sigma^{6}$ term with positive coefficient $f$ as the minimal extension of the model to stabilize the system.

TABLE I: For three flavors, with $\gamma>0$ and $\lambda=0$ : locations of $\mathrm{A}$, the critical point, $\mathrm{B}$, the critical end point, $\mathrm{C}$, the triple point, and $\mathrm{D}$, the tri-critical point, in Fig. 11 The point $\mathrm{D}$ appears for $\gamma>(\sqrt{\beta / b}) c / 3$. For two flavors with $b<0$ and $\lambda>0$ : locations of $\mathrm{E}$ and $\mathrm{F}$ in Fig. 3 Here $c^{\prime} \equiv \sqrt{c^{2}+16 b \gamma^{2} / \beta}$ and $b^{\prime} \equiv b-4 \lambda^{2} / \beta$.

\begin{tabular}{|c|c|c|}
\hline & $a$ & $\alpha$ \\
\hline \hline $\mathrm{A}$ & $c^{2} /(3 b)+2 \gamma^{2} / \beta$ & $-\beta c^{3} /\left(27 \gamma b^{2}\right)$ \\
$\mathrm{B}$ & $2 c^{2} /(9 b)+2 \gamma^{2} / \beta$ & 0 \\
$\mathrm{C}$ & $2 c^{2} /(9 b)$ & $\sqrt{\beta / b}(c /(3 \sqrt{b})+\gamma / \sqrt{\beta})^{2}$ \\
$\mathrm{D}$ & $\left(c+c^{\prime}\right) c /(8 b)-\gamma^{2} / \beta$ & $\left(c+c^{\prime}\right) \gamma /(2 b)$ \\
\hline $\mathrm{E}$ & $3 b^{2} /(16 f)$ & 0 \\
$\mathrm{~F}$ & $3 b^{\prime}\left(b^{\prime}+16 \lambda^{2} / \beta\right) /(16 f)$ & $3 \lambda b^{\prime} /(2 f)$ \\
\hline
\end{tabular}

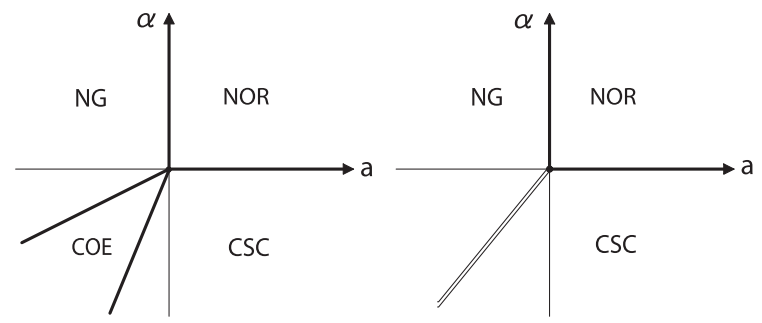

FIG. 2: Phase strucuture in the two-flavor system for $b>0$ and $\lambda>0$. Left: Case with the tetra-critical point $\left(\frac{1}{2} \sqrt{b \beta}>\right.$ $\lambda>0$ ). The second order line between NG and COE (CSC and COE $)$ is characterized by $\alpha=2 a \lambda / b(\alpha=a \beta /(2 \lambda))$. Right: Case with the bi-critical point $\left(\lambda>\frac{1}{2} \sqrt{b \beta}\right)$. The first order line between NG and CSC is characterized by $\alpha=a \sqrt{\beta / b}$.

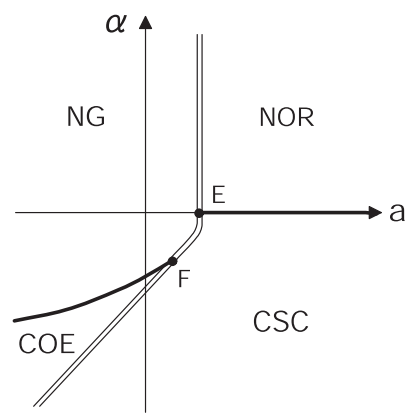

FIG. 3: Phase strucuture of the two-flavor system with $b<0$ and $\lambda>0$. The second order line between NG and COE is $\alpha=\lambda\left(b-\sqrt{b^{2}-4 f a}\right) / f$. The first order line between CSC and $\mathrm{COE}$ is at $\alpha=\frac{\beta}{2 \lambda}\left[a-3\left(b-4 \lambda^{2} / \beta\right)^{2} /(16 f)\right]$. The first order line between $\mathrm{NG}$ and CSC is at $\alpha^{2}=\beta\left[6 b f a+\left(b^{2}-\right.\right.$ $\left.4 f a)^{3 / 2}-b^{3}\right] /\left(6 f^{2}\right)$.

We first consider $b>0$ in Eq. (6), neglecting the $\sigma^{6}$ term in finding the qualitative phase structure [16]. For $\lambda=0$, the boundaries of the four phases are characterized by second order lines at $\alpha=0$ and $a=0$ with a tetracritical point at $\alpha=a=0$. With the repulsive $d^{2} \sigma^{2}$ term $(\lambda>0)$, the area of the coexistence phase decreases, as shown on the left of Fig. [2 For $\lambda>\frac{1}{2} \sqrt{b \beta}$, the coexistence region disappears, a first order interface between CSC and NG appears at $\alpha=a \sqrt{\beta / b}$, and $a=\alpha=0$ becomes a bi-critical point, as shown in the right of Fig 2

Next we consider $b<0$; here the $\sigma^{6}$ term plays an essential role. For $\lambda=0$, the four phases are separated by a second order line at $\alpha=0$ and a first order line at $a=3 b^{2} /(16 f)$. With a repulsive $d^{2} \sigma^{2}$ term $(\lambda>0)$ the coexistence phase shrinks and gradually fades away as $\lambda \rightarrow \infty$. Moreover, a new first order line between NG and CSC appears and grows as $\lambda$ increases. This situation is shown in Fig. 3 The locations of the critical end points $\mathrm{E}$ and $\mathrm{F}$ are given in Table I. Such a phase structure was previously noted in 17] which used the random matrix model and in 14] which used the NJL model; our modelindependent analysis is consistent with these results.

The mapping of the phase diagrams in the $(a, \alpha)$ plane 
to the $(T, \mu)$ plane is a dynamical question which we cannot address within the GL theory. Nevertheless, in Fig. 4. we draw a speculative phase structure with two light quarks and a medium-heavy quark. There are two critical points in the figure: one near the vertical axis driven by finite $\mu$ (as seen in the two-flavor case), and a new critical point near the horizontal axis driven by the axial anomaly (as seen in the three-flavor case). With decreasing strange quark mass, $m_{s}$, the higher critical point approaches the vertical axis, while as $m_{s}$ increases the lower critical point approaches horizontal axis. The chiral transition is a crossover in the direction of both high $T$ and high $\mu$. Whether this scenario is realized or not should be eventually checked by first principles QCD simulations.

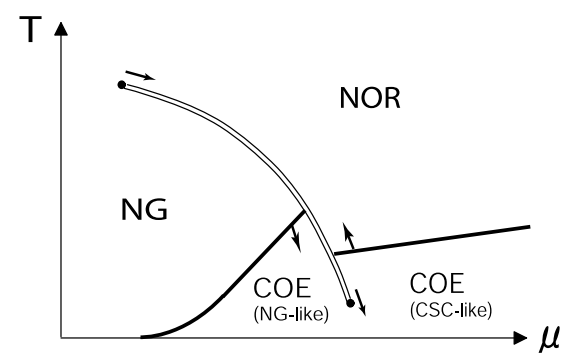

FIG. 4: Schematic phase structure with two light quarks (up and down) and a medium heavy quark (strange). The arrows show how the critical points and the phase boundaries move as the strange-quark mass increases toward the two-flavor limit.

In summary, we find that the QCD axial anomaly acts as an external field for the chiral condensate under the influence of the diquark condensate. The first order chiral transition is changed to a crossover for a large diquark condensate, and a new critical point driven by the axial anomaly emerges in the QCD phase diagram. Precise location this point is a future task for phenomenological models and lattice QCD simulations. Our schematic phase diagram would be made more realistic by including effects, such as finite quark masses, charge neutrality, $\beta$ equilibrium, and thermal gluon fluctuations 9,18 . Open questions include whether the new critical point would survive in an inhomogeneous Fulde-Ferrell-LarkinOvchinnikov (FFLO) state, and how the COE phase at low $T$ and $\mu$, Fig 4 is affected by quark confinement.

We thank S. Digal, K. Itakura, T. Matsuura, and K. Fukushima for helpful comments. This research was supported in part by the Grants-in-Aid of the Japanese Ministry of Education, Culture, Sports, Science, and Technology (No. 15540254), and in part by NSF Grant PHY
03-55014. Author GB thanks the University of Tokyo for kind hospitality and, in particular, the CNS for support.

[1] Reviewed in K. Rajagopal and F. Wilczek, in Handbook of $Q C D$, ed. M. Shifman (World Scientific, Singapore, 2001); M.G. Alford, Ann. Rev. Nucl. Part. Sci. 51, 131 (2001).

[2] K. Yagi, T. Hatsuda and Y. Miake, Quark-Gluon Plasma, Cambridge Univ. Press (Cambridge, 2005).

[3] Reviewed in M. Buballa, Phys. Rept. 407, 205 (2005).

[4] M. Sigrist and K. Ueda, Rev. Mod. Phys. 63, 239 (1991).

[5] T. Schäfer and F. Wilczek, Phys. Rev. Lett. 82, 3956 (1999).

[6] R.D. Pisarski and F. Wilczek, Phys. Rev. D29, 338 (1984).

[7] K. Iida and G. Baym, Phys. Rev. D 63, 074018 (2001); ibid. 66, 059903(E) (2002).

[8] I. Giannakis and H.-c. Ren, Nucl. Phys. B 669, 462 (2003).

[9] K. Iida, T. Matsuura, M. Tachibana and T. Hatsuda, Phys. Rev. D 71, 054003 (2005).

[10] The $\lambda_{3}$ term originates from the polynomial structure, $\epsilon_{i j k} \epsilon_{i^{\prime} j^{\prime} k^{\prime}} \Phi_{i i^{\prime}} \Phi_{j j^{\prime}}\left(d_{L} d_{R}^{\dagger}\right)_{k k^{\prime}}$. See D. T. Son and M. A. Stephanov, Phys. Rev. D 61, 074012 (2000).

[11] T. Schäfer, Phys. Rev. D 65, 094033 (2002). R. Rapp, T. Schäfer, E. V. Shuryak and M. Velkovsky, Ann. Phys. (N.Y.) 280, 35 (2000).

[12] N. Yamamoto, M. Tachibana, T. Hatsuda and G. Baym, in preparation.

[13] A similar critical point (CP) at low temperature has been derived in [14] using the two-flavor NJL model with scalar and vector type four-fermion interactions. Since the axial anomaly does not produce a triple boson coupling in two flavors, the origin of their $\mathrm{CP}$ is not related to ours.

[14] M. Kitazawa, T. Koide, T. Kunihiro and Y. Nemoto, Prog. Theor. Phys. 108, 929 (2002).

[15] M. Asakawa and K. Yazaki, Nucl. Phys. A 504, 668 (1989). A. Barducci et al., Phys. Lett. B231, 463 (1989); M. Stephanov, K. Rajagopal and E. Shuryak, Phys. Rev. Lett. 81, 4816 (1998); Y. Hatta and T. Ikeda, Phys. Rev. D 67, 014028 (2003).

[16] The system is equivalent to an anisotropic antiferromagnet such as $\mathrm{GdAlO}_{3}$. See, e.g., P. M. Chaikin and T. C. Lubensky, Principles of Condensed Matter Physics, Cambridge Univ. Press (Cambridge, 1995), Chap. 4.6.4.

[17] B. Vanderheyden and A. D. Jackson, Phys. Rev. D 62 094010 (2000). However, the random matrix model does not include the physics of the suppression of the density of states at the Fermi surface leading to $\lambda>0$, and thus their phase structure is not obviously related to ours.

[18] T. Matsuura, K. Iida, T. Hatsuda, and G. Baym, Phys. Rev. D 69, 074012 (2004); I. Giannakis, D.-f. Hou, H.c. Ren, and D. H. Rischke, Phys. Rev. Lett. 93, 232301 (2004). 